\title{
Minimum-weight combinatorial structures under random cost-constraints
}

\author{
Alan Frieze* Wesley Pegden ${ }^{\dagger}$ \\ Department of Mathematical Sciences \\ Carnegie Mellon University \\ Pittsburgh PA 15213, U.S.A. \\ alan@random.math.cmu.edu wes@math.cmu.edu \\ Gregory B. Sorkin \\ Department of Mathematics \\ The London School of Economics and Political Science \\ London WC2A 2AE, U.K. \\ g.b.sorkin@lse.ac.uk \\ Tomasz Tkocz \\ Department of Mathematical Sciences \\ Carnegie Mellon University \\ Pittsburgh PA 15213, U.S.A. \\ ttkocz@andrew.cmu.edu
}

Submitted: Feb 27, 2020; Accepted: Nov 4, 2020; Published: Jan 29, 2021

(c) The authors

\begin{abstract}
Recall that Janson showed that if the edges of the complete graph $K_{n}$ are assigned exponentially distributed independent random weights, then the expected length of a shortest path between a fixed pair of vertices is asymptotically equal to $(\log n) / n$. We consider analogous problems where edges have not only a random length but also a random cost, and we are interested in the length of the minimumlength structure whose total cost is less than some cost budget. For several classes of structures, we determine the correct minimum length structure as a function of the cost-budget, up to constant factors. Moreover, we achieve this even in the more
\end{abstract}

\footnotetext{
${ }^{*}$ Research supported in part by NSF grant DMS1661063.

${ }^{\dagger}$ Research supported in part by NSF grant DMS1363136

${ }^{\ddagger}$ Research supported in part by the Collaboration Grants from the Simons Foundation and NSF grant DMS1955175
} 
general setting where the distribution of weights and costs are arbitrary, so long as the density $f(x)$ as $x \rightarrow 0$ behaves like $c x^{\gamma}$ for some $\gamma \geqslant 0$; previously, this case was not understood even in the absence of cost constraints. We also handle the case where each edge has several independent costs associated to it, and we must simultaneously satisfy budgets on each cost. In this case, we show that the minimum-length structure obtainable is essentially controlled by the product of the cost thresholds.

Mathematics Subject Classifications: 05C80, 05C85, 90C27

\section{Introduction}

Let the edges of the complete graph be given independent random edge weights $w(e)$ and a random cost $c(e)$ for $e \in E\left(K_{n}\right)$. We are interested in the problem of estimating the minimum weight of a combinatorial structure $S$ where the total cost of $S$ is bounded by some value $C$. More generally, we allow $r \operatorname{costs} \mathbf{c}(e)=\left(c_{i}(e), i=1,2, \ldots, r\right)$ for each edge. The distribution of weights $w(e)$ will be independent copies of $Z_{E}^{\alpha}$ where $Z_{E}$ denotes the exponential rate one random variable and $\alpha \leqslant 1$. The distribution of $\operatorname{costs} c_{i}(e)$ will be independent copies of $Z_{E}^{\beta}$ where $\beta \leqslant 1$. In Section 6 we will see that, since we are allowing powers of exponentials, a simple coupling argument will allow us to model a very general class of independent weights and costs, where we require just that the densities satisfy $f(x) \approx c x^{\gamma}, \gamma \geqslant 0$ as $x \rightarrow 0$; here we mean that $c x^{\gamma} / f(x) \rightarrow 1$ as $x \rightarrow 0$.

Suppose we are given cost budgets of $\mathbf{C}=\left(C_{i}, i=1,2, \ldots, r\right)$ and we consider the following problem: let $\mathcal{S}$ denote some collection of combinatorial strutures such as paths, matchings, Hamilton cycles, we would like to solve

$$
\operatorname{Opt}(\mathcal{S}, \mathbf{C}) \text { : Minimise } w(S) \text { subject to } S \in \mathcal{S} \text { and } c_{i}(S) \leqslant C_{i}, i=1,2, \ldots, r,
$$

and let

$$
w^{*}(\mathbf{C}) \text { denote the minimum value in } \operatorname{Opt}(\mathcal{S}, \mathbf{C}) .
$$

We remark that Frieze and Tkocz [9], [10] have considered finding minimum weight spanning trees or arborescences in the context of a single cost constraint and uniform $[0,1]$ weights and costs. I.e. the case where $\mathcal{S}$ is the set of spanning trees of $K_{n}$ and the case where $\mathcal{S}$ is the set of spanning arborescences of $\vec{K}_{n}$. In these cases, they obtain asymptotically optimal estimates for those problems, whereas for the problems in the present paper we have only obtained estimates that are correct to within a constant factor.

The first problem we study involves paths, here denoted as minimum weight paths for consistency with the remainder of the paper. Let $\mathcal{P}(i, j)$ denote the set of paths from vertex $i$ to vertex $j$ in $K_{n}$.

Constrained Minimum Weight Path (CMWP): $\operatorname{Opt}(\mathcal{P}(1, n), \mathbf{C})$.

Without the constraint $\mathbf{c}(P) \leqslant \mathbf{C}$, there is a beautiful result of Janson [13] that gives a precise value for the expected minimum weight of a path, when the $w(e)$ 's are independent exponential mean one. With the constraints, we are only able to estimate the expected minimum weight up to a constant (but can do so for a more general class of distributions). 
Throughout the paper we let

$$
\Upsilon=\prod_{i=1}^{r} C_{i}
$$

be the product of the cost thresholds. Our results show that for the structures we consider, this product of the cost thresholds controls the dependency of the minimum-weight structure on the vector of cost constraints. In particular, for the minimum weight path problem, we have:

Theorem 1. If $\frac{n \Upsilon^{1 / \beta}}{\log ^{r / \beta} n} \rightarrow \infty$ and $C_{i} \leqslant 10 \log n, i=1,2, \ldots, r$ then w.h.p.

$$
w^{*}(\mathbf{C})=\Theta\left(\frac{\log ^{r \alpha / \beta+1} n}{n^{\alpha} \Upsilon^{\alpha / \beta}}\right) .
$$

$A=\Theta(B)$ denotes $A=O(B)$ and $B=O(A)$. And here, the hidden constants depend only on $r, \alpha, \beta$.

For the unconstrained problem, see Hassin and Zemel [11], Janson [13] and Bhamidi and van der Hofstad [2], [3].

Now consider the case of perfect matchings in the complete bipartite graph $K_{n, n}$. Let $\mathcal{M}_{2}$ denote the set of perfect matchings in $K_{n, n}$.

Constrained Assigment Problem (CAP): $\operatorname{Opt}\left(\mathcal{M}_{2}, \mathbf{C}\right)$.

Theorem 2. If $\Upsilon^{1 / \beta} \gg n^{r / \beta-1} \log n^{1}$ and $C_{i} \leqslant n, i=1,2, \ldots, r$ then w.h.p.

$$
w^{*}(\mathbf{C})=\Theta\left(\frac{n^{1+r \alpha / \beta-\alpha}}{\Upsilon^{\alpha / \beta}}\right) \text {. }
$$

We note that requiring a lower bound on $\Upsilon$ is necessary in Theorem 2 . Indeed, if $\Upsilon^{1 / \beta} \leqslant e^{-(r+1)} \beta n^{r / \beta-1}$ then the optimization problem is infeasible w.h.p. To see this we bound the expected number of feasible solutions as follows: let $Z_{1}, Z_{2}, \ldots, Z_{r}$ be independent sums of $n$ independent copies of $Z_{E}^{1 / \beta}$. Then,

$$
n ! \prod_{i=1}^{r} \mathbb{P}\left(Z_{i} \leqslant C_{i}\right) \leqslant n ! \prod_{i=1}^{r} \frac{C_{i}^{n / \beta}}{\beta^{n} n ! n^{n(1 / \beta-1)}} \leqslant\left(\frac{e^{r} \Upsilon^{1 / \beta}}{\beta n^{r / \beta-1}}\right)^{n}=o(1) .
$$

We use Lemma 7 here to bound $\mathbb{P}\left(Z_{i} \leqslant C_{i}\right)$. We note that a problem similar to this was studied by Arora, Frieze and Kaplan [1] with respect to the worst-case.

Now consider the case of perfect matchings in the complete graph $K_{n}$. Let $\mathcal{M}_{1}$ denote the set of perfect matchings in $K_{n}$.

Constrained Matching Problem (CMP) $\operatorname{Opt}\left(\mathcal{M}_{1}, \mathbf{C}\right)$.

Theorem 3. If $\Upsilon^{1 / \beta} \gg n^{r-1} \log n$ and $C_{i} \leqslant n, i=1,2, \ldots, r$ then w.h.p.

$$
w^{*}(\mathbf{C})=\Theta\left(\frac{n^{1+r \alpha / \beta-\alpha}}{\Upsilon^{\alpha / \beta}}\right) \text {. }
$$

\footnotetext{
${ }^{1}$ Here $A=A(n) \gg B=B(n)$ if $A / B \rightarrow \infty$ as $n \rightarrow \infty$.
} 
Now consider the following set of problems: Minimum Spanning Tree (MST), Minimum Spanning Arborescence (MSA), Travelling Salesperson Problem (TSP). Let $\mathcal{T}$ denote the set of Hamilton cycles in $K_{n}$ or the set of directed Hamilton cycles in $\vec{K}_{n}$.

Constrained Spanning Tree/Arborescence/Travelling Salesperson Problem $(\mathbf{C T S P}+) \operatorname{Opt}(\mathcal{T}, \mathbf{C})$.

Theorem 4. If $\Upsilon^{1 / \beta} \gg n^{r-1} \log n$ and and $C_{i} \leqslant n, i=1,2, \ldots, r$ then w.h.p.

$$
w^{*}(\mathbf{C})=\Theta\left(\frac{n^{1+r \alpha / \beta-\alpha}}{\Upsilon^{\alpha / \beta}}\right)
$$

\section{Structure of the paper}

We prove the above theorems in their order of statement. The upper bounds are proved as follows: we consider the random graph $G_{n, p}$ (or bipartite graph $G_{n, n, p}$ or digraph $D_{n, p}$ ) for suitably chosen $p$ associated with the random costs. We then seek minimum weight objects contained in these random graphs. The definition of $p$ is such that objects, if they exist, automatically satisfy the cost constraints. For minimum weight paths we adapt the methodology of [13]. For the remaining problems we use theorems in the literature stating the high probability existence of the required objects when each vertex independently chooses a few (close) random neighbors.

In Section 6 we consider more general distributions. We are able to extend the above theorems under some extra assumptions about the $C_{i}$.

\section{CSP}

\subsection{Upper Bound for CSP}

In the proof of the upper bound, we first consider weights $\widehat{w}(e)$ where the $\widehat{w}(e)$ are independent exponential mean one random variables. The costs will remain independent copies of $Z_{E}^{\beta}$. We will then use Holder's inequality to obtain the final result.

$$
3.2 \frac{\log ^{2+r / \beta} n}{n} \leqslant \Upsilon^{1 / \beta} \text { and } C_{i} \leqslant 10 \log n, i=1,2, \ldots, r
$$

Suppose now that we let $L=10 \log n$ and

$$
E_{0}=\left\{e: c_{i}(e) \leqslant \frac{C_{i}}{L}, i=1,2, \ldots r\right\} .
$$

The proof in this case goes as follows:

(i) We search for short paths that only use edges in $E_{0}$ and note that the graph $\left([n], E_{0}\right)$ is distributed as $G_{n, p}$.

(ii) Observe that any path using fewer than $L$ edges of $E_{0}$ automatically satifies the cost constraints. 
(iii) A simple calculation shows that w.h.p. the number of edges between a set $S$ of size $k$ and the remaining vertices is close to the expectation $k(n-k) p$ for all sets of vertices $S$, see (3) and (4).

(iv) We run Dijkstra's algorithm for finding shortest (now minimum weight) paths from vertex 1. We use Janson's argument [13] to bound the distance to the $m=n / 3$ closest vertices $V_{1}$. We need the claim in item (iii) here.

(v) We repeat (iv), starting from vertex set $n$, to obtain the $m=n / 3$ closest vertices $V_{2}$. If $V_{1} \cap V_{2} \neq \emptyset$ we will have found a path of low enough weight, otherwise we claim that w.h.p. there will be a low enough weight edge joining $V_{1}, V_{2}$.

(vi) We then argue that the trees constructed by the Dijkstra algorithm are close to being Random Recursive Trees and we can easily bound their height. Showing that we can use item (ii).

(vii) We finally use Holder's inequality to switch from $\widehat{w}$ to $w$.

We first bound the value of $\mathbb{P}\left(e \in E_{0}\right)$.

$$
p=\mathbb{P}\left(e \in E_{0}\right)=\prod_{i=1}^{r}\left(1-\exp \left\{-\left(\frac{C_{i}}{3 L}\right)^{1 / \beta}\right\}\right),
$$

where $e$ is an arbitrary edge.

We note that if $0<x \leqslant 1$ then $x / 2 \leqslant 1-e^{-x} \leqslant x$. This implies that

$$
\frac{\Upsilon^{1 / \beta}}{2^{r}(3 L)^{r / \beta}} \leqslant p \leqslant \frac{\Upsilon^{1 / \beta}}{(3 L)^{r / \beta}}
$$

We consider the random graph $G_{n, p}$ where edges have weight given by $\widehat{w}$ and $\operatorname{costs} c_{i}(e) \leqslant$ $C_{i} / 3 L, i=1,2, \ldots, r$. We modify Janson's argument [13].

We now deal with item (iii). We observe that w.h.p. for every set $S$ of size $k$, $e(S: \bar{S}) \approx k(n-k) p$ where $e(S: T)$ is the number of edges $\{v, w\}$ with one end in $S$ and the other in $T$. We only need to check the claim for $|S| \leqslant n / 2$. Let $\varepsilon=\frac{1}{\log ^{1 / 3} n}$ and

$$
\mathcal{E}_{S}=\{e(S: \bar{S}) \notin(1 \pm \varepsilon) k(n-k) p\} \text { and } \mathcal{E}=\bigcup_{|S| \leqslant n / 2} \mathcal{E}_{S}
$$


Then, using the Chernoff bounds for the binomial distribution,

$$
\begin{aligned}
\mathbb{P}(\mathcal{E}) & \leqslant \sum_{k=1}^{n / 2}\left(\begin{array}{l}
n \\
k
\end{array}\right) \mathbb{P}(\operatorname{Bin}(k(n-k), p) \notin(1 \pm \varepsilon) k(n-k) p) \\
& \leqslant 2 \sum_{k=1}^{n / 2}\left(\frac{n e}{k}\right)^{k} e^{-\varepsilon^{2} k(n-k) p / 3} \\
& =2 \sum_{k=1}^{n / 2}\left(\frac{n e^{1-\varepsilon^{2}(n-k) p / 3}}{k}\right)^{k} \\
& \leqslant 2 \sum_{k=1}^{n / 2}\left(\frac{n e^{-\Omega\left(\log ^{4 / 3} n\right)}}{k}\right) \\
& =o(1),
\end{aligned}
$$

where we have used (2) to get the last inequality.

We now continue with item (iv). We set $S_{1}=\{1\}$ and $d_{1}=0$ and consider running Dijkstra's algorithm [6]. At the end of Step $k$, we will have computed $S_{k}=$ $\left\{1=v_{1}, v_{2}, \ldots, v_{k}\right\}$ and $0=d_{1}, d_{2}, \ldots, d_{k}$ where $d_{i}$ is the minimum weight of a path from 1 to $i, i=1,2, \ldots, k$. Let there be $\nu_{k}$ edges from $S_{k}$ to $[n] \backslash S_{k}$. Arguing as in [13] we see that $d_{k+1}-d_{k}=Z_{k}$ where $Z_{k}$ is the minimum of $\nu_{k}$ independent exponential mean one random variables. Also, the memoryless property of the exponential distribution implies that $Z_{k}$ is independent of $d_{k}$. It follows that for $k<n / 2$,

$$
\begin{aligned}
\mathbb{E}\left(d_{k} \mid \neg \mathcal{E}\right)=\mathbb{E}\left(\sum_{i=1}^{k} \frac{1}{\nu_{i}} \mid \neg \mathcal{E}\right)=\sum_{i=1}^{k} \frac{1+o(1)}{i(n-i) p} & =\frac{1+o(1)}{n p} \sum_{i=1}^{k}\left(\frac{1}{i}+\frac{1}{n-i}\right) \\
& =\frac{1+o(1)}{n p}\left(H_{k}+H_{n-1}-H_{n-k+1}\right),
\end{aligned}
$$

where $H_{k}=\sum_{i=1}^{k} \frac{1}{i}$.

By the same token,

$$
\operatorname{Var}\left(d_{k} \mid \neg \mathcal{E}\right)=\sum_{i=1}^{k} \operatorname{Var}\left(Z_{i} \mid \neg \mathcal{E}\right)=\sum_{i=1}^{k} \frac{1+o(1)}{(i(n-i) p)^{2}}=O\left((n p)^{-2}\right) .
$$

We only pursue the use of Dijkstra's algoritm from vertex 1 for $m=n / 3$ iterations. It follows from (5) and (6) and the Chebyshev inequality that we have w.h.p.

$$
d_{m} \approx \frac{\log n}{n p}
$$

We next deal with item (vi). The tree built by Dijkstra's algorithm is close in distribution to a random recursive tree i.e. vertex $v_{k+1}$ attaches to a near uniformly random member 
of $\left\{v_{1}, v_{2}, \ldots, v_{k}\right\}$. Indeed, assuming $\mathcal{E}$ does not occur,

$$
\mathbb{P}\left(v_{k+1} \text { attaches to } v_{i}\right)=\frac{e\left(v_{i}: \bar{S}_{k}\right)}{\nu_{k}} \leqslant \frac{(1+\varepsilon)(n-1) p}{(1-\varepsilon) k(n-k) p} .
$$

Hence, if $T$ is the tree constructed in the first $m$ rounds of Dijkstra's algorithm, then

$$
\begin{aligned}
\mathbb{P}(\text { height }(T) \geqslant L) & \leqslant \sum_{1<t_{1}<\cdots<t_{L}<m} \prod_{i=1}^{L} \frac{3(1+\varepsilon)}{2(1-\varepsilon) t_{i}} \\
& \leqslant \frac{1}{L !}\left(\frac{3(1+\varepsilon)}{2(1-\varepsilon)}\right)^{L}\left(\sum_{i=1}^{n} \frac{1}{i}\right)^{L} \\
& \leqslant\left(\frac{3(\log n+1) e^{1+o(1)}}{2 L}\right)^{L}=o(1) .
\end{aligned}
$$

It follows from (2), (7) and (8) that w.h.p., for every $v \in V_{1}=S_{m}$, there exists a path $P$ from 1 to $v$ of weight at most

$$
\lambda \approx \lambda_{0}=\frac{\log n}{n p} \lesssim \frac{30^{r / \beta} \log ^{r / \beta+1} n}{n \Upsilon^{1 / \beta}}
$$

and $\operatorname{costs} c_{i}(P) \leqslant L C_{i} / 3 L \leqslant C_{i} / 3 .^{2}$

We now deal with item (v). We next consider applying Dijkstra's algorithm to find a minimum weight path from vertex $n$ to other vertices. Using the same argument as above, we see that we can find $m$ vertices $V_{2}$ that are within distance $\lambda_{0}$ of vertex $n$. If $V_{1} \cap V_{2} \neq \emptyset$ then we have found a path of weight at most $2 \lambda_{0}$ between vertex 1 and vertex $n$.

If $V_{1}, V_{2}$ are disjoint then w.h.p. there is an edge of weight $20 / n p$ between them. Indeed,

$$
\mathbb{P}\left(\exists V_{1}, V_{2} \text { with no such edge }\right) \leqslant\left(\begin{array}{c}
n \\
m
\end{array}\right)^{2}\left(e^{-20 / n p}\right)^{n^{2} / 9}=o(1) .
$$

This yields a path $P$ with

$$
\begin{aligned}
& \widehat{w}(P) \leqslant 2 \lambda_{0}+\frac{20}{n p} \leqslant \frac{3 \cdot 30^{r / \beta} \log ^{r / \beta+1} n}{n \Upsilon^{1 / \beta}} . \\
& c_{i}(P) \leqslant \frac{2 C_{i}}{3}+\frac{C_{i}}{3}=C_{i}, \quad i=1,2, \ldots, r .
\end{aligned}
$$

(Here we have used $C_{i} \geqslant \Upsilon^{1 / \beta} / L^{r-1} \gg p$.)

We now deal with item (vii). We use Holder's inequality to yield

$$
w(P)=\sum_{e \in P} \widehat{w}(e)^{\alpha} \leqslant\left(\sum_{e \in P} \widehat{w}(e)\right)^{\alpha} L^{1-\alpha}=O\left(\frac{\log ^{r \alpha / \beta+1} n}{n^{\alpha} \Upsilon^{\alpha / \beta}}\right) .
$$

This completes the proof of Theorem 1 for this case.

\footnotetext{
${ }^{2}$ Here we write $A=A(n) \lesssim B=B(n)$ if $A \leqslant(1+o(1)) B$.
} 


\section{$3.3 \frac{3 \omega \log ^{r / \beta} n}{n} \leqslant \Upsilon^{1 / \beta} \leqslant \frac{\log ^{r / \beta+2} n}{n}$ and $C_{i} \leqslant 10 \log n, i=1,2, \ldots, r$}

The proof is similar to that of Section 3.2, but requires some changes in some places. The problem is that we cannot now assume the non-occurrence of $\mathcal{E}$. Other than this, the proof will follow the same strategy. Our problem therefore is to argue that w.h.p. $e\left(S_{k}: \bar{S}_{k}\right)$ is sufficiently large.

(a) We now have to keep track of the size of $e\left(S_{k}: \bar{S}_{k}\right)$ as a random process. This is equation (12).

(b) The term $\eta_{k}$ is the number of edges between $v \notin S_{k}$ and $S_{k}$. We don't want this to be large, as it reduces $e\left(S_{k+1}: \bar{S}_{k+1}\right)$. So, we do not add vertices to $S_{k}$ if $\eta_{k} \geqslant 2 n p$, which only happends rarely.

(c) Finally, we have to work harder in the case where $V_{1}, V_{2}$ are disjoint. We need to use edges of slightly higher cost in order to get a low weight edge in $e\left(V_{1}: V_{2}\right)$.

Let $p$ be as in (1) where $L=20 \log n$. Note that from (2) we see that

$$
p \leqslant \frac{\log ^{2} n}{n}
$$

We again consider the random graph $G_{n, p}$ where edges have weight given by $\widehat{w}$ and costs at most $C_{i} / 3 L$ and again modify Janson's argument [13]. We also restrict our search for paths, avoiding vertices of high degree.

We set $S_{1}=\{1\}$ and $d_{1}=0$. At the end of Step $k$ we will have computed $S_{k}=$ $\left\{1=v_{1}, v_{2}, \ldots, v_{k}\right\}$ and $0=d_{1}, d_{2}, \ldots, d_{k}$ where $d_{i}$ is the minimum weight of a path from 1 to $i, i=1,2, \ldots, k$. Let there be $\nu_{k}$ edges from $S_{k}$ to $[n] \backslash S_{k}$. We cannot rely on $\mathcal{E}$ of (4) not to occur and so we need to modify the argument here.

Assumption: $1 \leqslant k \leqslant n_{0}=1 / 3 p$

Modification: if our initial choice $v$ for $v_{k+1}$ satisfies $e\left(v: \bar{S}_{k}\right) \geqslant 2 n p$ then we reject $v$ permanently from the construction of paths from vertex 1 .

The initial aim is roughly the same, we want to show that w.h.p.

$$
\sum_{\ell \leqslant k} \nu_{\ell} \geqslant(1-o(1)) k n p
$$

For $v \notin S_{k}$, let $\eta_{k, v}=e\left(S_{k}:\{v\}\right)$ and $\eta_{k}=\eta_{k, v_{k+1}}$. Then, w.h.p.

$$
\nu_{k+1} \geqslant \nu_{k}-\eta_{k}+B_{k} \text { where } B_{k}=\operatorname{Bin}\left(n_{1}, p\right) 1_{B i n(n, p) \leqslant 2 n p},
$$

where $n_{1}=n-2 n_{0}$.

The binomials are independent here. This is because the edges between $v_{k+1}$ and $\bar{S}_{k}$ have not been exposed by the algorithm to this point. The number of trials $n_{1}$ comes from the following: we know from the Chernoff bounds that

$$
\mathbb{P}(\operatorname{Bin}(n, p) \geqslant 2 n p) \leqslant e^{-n p / 3} \text {. }
$$


It follows from the Markov inequality that w.h.p. there are at most $n e^{-n p / 4}$ instances where the modification is invoked. This means that w.h.p. the initial choice for $v_{k}$ has at least $n-n_{0}-n e^{-n p / 4} \geqslant n_{1}$ possible neighbors. We now define

$$
S_{k}=\sum_{\ell=1}^{k} B_{k}
$$

We need a lower bound for $B_{k}$ and an upper bound for $\eta_{k}$. We next observe that if

$$
\varepsilon=(n p)^{-1 / 3}
$$

then

$\mathbb{P}\left(B_{k} \leqslant(1-\varepsilon) n p\right)=\mathbb{P}\left(\operatorname{Bin}\left(n_{1}, p\right) \geqslant 2 n p\right)+\mathbb{P}\left(\operatorname{Bin}\left(n_{1} \leqslant(1-\varepsilon) n p\right)\right) \leqslant(1+o(1)) e^{-\varepsilon^{2} n p / 3}$.

It follows that if $k_{0}=\min \left\{n_{0}, e^{\varepsilon^{2} n p / 4}\right\}$ then w.h.p.

$$
\mathbb{P}\left(\exists 0 \leqslant k \leqslant k_{0}: B_{k} \leqslant(1-\varepsilon) n p\right) \leqslant(1+o(1)) k_{0} e^{-\varepsilon^{2} n p / 3} \leqslant e^{-\varepsilon^{2} n p / 12} .
$$

For $k \geqslant k_{0}$, we use the fact that $S_{k}$ is the sum of bounded random variables. Hoeffding's inequality [12] gives that

$$
\mathbb{P}\left(S_{k} \leqslant \mathbb{E}\left(S_{k}\right)-t\right) \leqslant \exp \left\{-\frac{2 t^{2}}{4 k n^{2} p^{2}}\right\}
$$

Now $\mathbb{E}\left(B_{k}\right) \geqslant(1-\varepsilon) n p$ and so putting $t=k^{2 / 3} n p$ we see that

$$
\mathbb{P}\left(S_{k} \leqslant(1-\varepsilon) k n p-k^{2 / 3} n p\right) \leqslant e^{-k^{1 / 3} / 2} .
$$

So

$$
\mathbb{P}\left(\exists k \geqslant k_{0}: S_{k} \leqslant(1-\varepsilon) k n p-k^{2 / 3} n p\right) \leqslant \sum_{k \geqslant k_{0}} e^{-k^{1 / 3} / 2}=o(1) .
$$

We next observe that

$$
\begin{aligned}
\mathbb{P}(\exists S:|S|=s \leqslant 1 / 3 p, e(S: S) \geqslant s+r) & \leqslant \sum_{s=1}^{1 / 3 p}\left(\begin{array}{c}
n \\
s
\end{array}\right)\left(\begin{array}{c}
s(s-1) / 2 \\
s+r
\end{array}\right) p^{s+r} \\
& \leqslant \sum_{s=1}^{1 / 3 p}\left(\frac{e^{2} n p}{2}\right)^{s}\left(\frac{s e p}{2}\right)^{r}
\end{aligned}
$$

Putting $r=s(n p)^{1 / 2}$, the RHS of (18) becomes

$$
\sum_{s=1}^{1 / 3 p}\left(\frac{e^{2} n p}{2}\left(\frac{s e p}{2}\right)^{(n p)^{1 / 2}}\right)^{s} \leqslant \sum_{s=1}^{1 / 3 p}\left(\frac{e^{2} n p}{2}\left(\frac{e}{6}\right)^{(n p)^{1 / 2}}\right)^{s}=o(1) .
$$


It follows that w.h.p.,

$$
\sum_{\ell=1}^{k} \eta_{\ell}=e\left(S_{k}\right) \leqslant 2\left((n p)^{1 / 2}+1\right) k .
$$

It then follows from (13) and (16) and (17) and (19) that w.h.p.

$$
\nu_{k} \geqslant(1-o(1)) k n p-2\left((n p)^{1 / 2}+1\right) k \geqslant(1-o(1)) k n p .
$$

Arguing as in [13] we see that $d_{k+1}-d_{k}=Z_{k}$ where $Z_{k}$ is the minimum of $\nu_{k}$ independent exponential mean one random variables. Also, $Z_{k}$ is independent of $d_{k}$. It follows that for $k<n$,

$$
\mathbb{E}\left(d_{k}\right)=\mathbb{E}\left(\sum_{i=1}^{k} \frac{1}{\nu_{i}}\right) \leqslant \sum_{i=1}^{k} \frac{1+o(1)}{i n p}=\frac{1+o(1)}{n p} \sum_{i=1}^{k} \frac{1}{i}=\frac{1+o(1)}{n p} H_{k},
$$

where $H_{k}=\sum_{i=1}^{k} \frac{1}{i}$.

By the same token,

$$
\operatorname{Var}\left(d_{k}\right)=\sum_{i=1}^{k} \operatorname{Var}\left(Z_{i}\right)=\sum_{i=1}^{k} \frac{1+o(1)}{(i n p)^{2}}=O\left((n p)^{-2}\right) .
$$

It follows from (21) and (22) and the Chebyshev inequality that w.h.p. we have $d_{n_{0}} \lesssim \frac{\log n}{n p}$. Let $V_{1}$ denote the $n_{0}$ vertices at this distance from vertex 1 .

We next consider applying Dijkstra's algorithm to find a minimum weight path from vertex $n$ to other vertices. Using the same argument as above, we see that we can find $n_{0}$ vertices $V_{2}$ that are within distance $\frac{(1+o(1)) \log n}{n p}$ of vertex $n$. If $V_{1} \cap V_{2} \neq \emptyset$ then we have found a path of weight at most $\frac{(2+o(1)) \log n}{n p}$ between vertex 1 and vertex $n$.

If $V_{1}, V_{2}$ are disjoint then we will use the edges

$$
E_{1}=\left\{e: c_{i}(e) \in\left[\frac{C_{i}}{L}, \frac{2 C_{i}}{L}\right], i=1,2, \ldots r\right\} .
$$

Given $e=\{x, y\} \in V_{1}: V_{2}$, then given the history of Dijkstra's algorithm so far, either $e \in E_{0}$ or we can say that

$$
\mathbb{P}\left(e \in E_{1} \mid e \notin E_{0}\right) \geqslant \mathbb{P}\left(e \in E_{1}\right)=\left(1-e^{-\left(2^{1 / \beta}-1\right) p^{1 / \beta}}\right)^{r} .
$$

For the equation in (23) we use

$$
\begin{aligned}
\mathbb{P}\left(p \leqslant Z_{E}^{\beta} \leqslant 2 p\right) & =\mathbb{P}\left(Z_{E}^{\beta} \geqslant p\right)\left(1-\mathbb{P}\left(Z_{E}^{\beta} \geqslant 2 p \mid Z_{E} \geqslant p\right)\right) \\
& =e^{-p^{1 / \beta}}\left(1-\frac{\mathbb{P}\left(Z_{E}^{\beta} \geqslant 2 p\right)}{\mathbb{P}\left(Z_{e}^{\beta} \geqslant p\right)}\right) \\
& =e^{-p^{1 / \beta}}\left(1-e^{-\left(2^{1 / \beta}-1\right) p^{1 / \beta}}\right) \\
& =e^{-p^{1 / \beta}}-e^{-(2 p)^{1 / \beta}} \geqslant \frac{\left(2^{1 / \beta}-1\right) p^{1 / \beta}}{2} .
\end{aligned}
$$


For the inequality in $(24)$ we use the fact that we now have $p \leqslant \frac{\log ^{2} n}{n}$.

Then we search for an edge in $E_{2}=\left\{e \in E_{1}: \widehat{w}(e) \leqslant 1 / n p\right\}$. And,

$$
\begin{aligned}
\mathbb{P}\left(E_{2} \cap\left(V_{1}: V_{2}\right)=\emptyset\right) & \leqslant\left(1-\left(\frac{\left(2^{1 / \beta}-1\right) p^{1 / \beta}}{2}\right)\left(1-e^{-1 / n p}\right)\right)^{1 / 9 p^{2}} \\
& \leqslant\left(1-\frac{\left(2^{1 / \beta}-1\right) p^{1 / \beta}}{2 n p}\right)^{1 / 9 p^{2}}=o(1) .
\end{aligned}
$$

This yields a path of weight at most $\frac{(2+o(1)) \log n}{n p}+\frac{1}{n p}=\frac{(2+o(1)) \log n}{n p}$.

We deal with the height of the Dijkstra trees. Let $T$ be the tree constructed by Dijkstra's algorithm and let $\xi_{i}, i \leqslant k$ denote the number of edges from $v_{i}$ to $V_{1} \backslash S_{i}$.

$$
\begin{aligned}
\mathbb{P}(\text { height }(T) \geqslant L) & \leqslant \mathbb{E}\left(\sum_{1<t_{1}<\cdots<t_{L}<n_{0}} \prod_{i=1}^{L} \frac{\xi_{t_{i}}}{\nu_{t_{i+1}-1}}\right) \\
& \leqslant \mathbb{E}\left(\sum_{1<t_{1}<\cdots<t_{L}<n_{0}} \prod_{i=1}^{L} \frac{2 n p}{\nu_{t_{i+1}-1}}\right) \\
& \leqslant \mathbb{E}\left(\frac{1}{L !}\left(\sum_{i=1}^{n_{0}} \frac{2 n p}{\nu_{i}}\right)^{L}\right) \\
& \leqslant o(1)+\frac{(2 e n p)^{L}}{(n p)^{L} L !}\left(\sum_{i=1}^{n_{0}} \frac{1+o(1)}{i}\right)^{L}=o(1),
\end{aligned}
$$

since $L \geqslant 20 \log n$.

The first $o(1)$ term in (25) is the probability that there is a small $\nu_{k}$ and this is covered by $(20)$.

It follows from the above that w.h.p. there exists a path $P$

$$
\text { where } \widehat{w}(P) \lesssim \frac{2 \log n}{n p} \text { and } c_{i}(P) \leqslant \frac{(2 L+2) C_{i}}{3 L}<C_{i}, i=1,2, \ldots, r \text {. }
$$

Arguing as for (11) we see that

$$
w(P) \leqslant \widehat{w}(P)^{\alpha} L^{1-\alpha}=O\left(\frac{\log ^{r \alpha / \beta+1} n}{n^{\alpha} \Upsilon^{\alpha / \beta}}\right) .
$$

\subsection{Lower Bound for CSP}

This is a straightforward use of the first moment method. Suppose that

$$
\Upsilon^{1 / \beta}=\frac{\omega \log ^{r / \beta} n}{n}, \quad L=\frac{\varepsilon \log ^{r \alpha / \beta+1} n}{n^{\alpha} \Upsilon^{\alpha / \beta}}
$$


where

$$
\varepsilon=\left(\alpha \beta^{r} e^{-2}\left(10\left(\frac{r}{\beta}+\frac{1}{\alpha}\right)\right)^{-(r / \beta+1 / \alpha)}\right)^{\alpha}
$$

then

$$
\begin{aligned}
& \mathbb{P}\left(\exists P: w(P) \leqslant L, c_{i}(P) \leqslant C_{i}, i=1,2, \ldots, r\right) \\
& \leqslant \sum_{k=1}^{n-2} n^{k-1}\left(\frac{L^{k / \alpha}}{\alpha^{k} k ! k^{k(1 / \alpha-1)}}\right) \prod_{i=1}^{r} \frac{C_{i}^{k / \beta}}{\beta^{k} k ! k^{k(1 / \beta-1)}} \\
& \leqslant \frac{1}{n} \sum_{k=1}^{n-1}\left(n \cdot \frac{e \varepsilon^{1 / \alpha} \log ^{r / \beta+1 / \alpha} n}{\alpha n k^{1 / \alpha} \Upsilon^{1 / \beta}} \cdot \frac{e \Upsilon^{1 / \beta}}{\beta^{r} k^{r / \beta}}\right)^{k} \\
& =\frac{1}{n} \sum_{k=1}^{n-1}\left(\frac{e^{2} \varepsilon^{1 / \alpha} \log ^{r / \beta+1 / \alpha} n}{\alpha \beta^{r} k^{r / \beta+1 / \alpha}}\right)^{k} \\
& =\frac{1}{n} \sum_{k=1}^{\frac{1}{2} \log n}\left(\frac{e^{2} \varepsilon^{1 / \alpha} \log { }^{r / \beta+1 / \alpha} n}{\alpha \beta^{r} k^{r / \beta+1 / \alpha}}\right)^{k}+\frac{1}{n} \sum_{k=\frac{1}{2} \log n}^{n-1}\left(\frac{e^{2} \varepsilon^{1 / \alpha} \log ^{r / \beta+1 / \alpha} n}{\alpha \beta^{r} k^{r / \beta+1 / \alpha}}\right)^{k} \\
& \leqslant \frac{1}{n} \sum_{k=1}^{\frac{1}{2} \log n}\left(\frac{\log n}{10(r / \beta+1 / \alpha) k}\right)_{k=\frac{1}{2} \log n}^{n-1} 10^{-k} \\
& =o(1) .
\end{aligned}
$$

Explanation for (28): we choose a path of length $k$ from 1 to $n$ in at most $n^{k-1}$ ways. Then we use Lemma $7 r+1$ times. Then we use the union bound.

\section{Upper Bounds}

\subsection{Upper Bound for CAP}

Let $G$ denote the subgraph of $K_{n, n}$ induced by the edges that satisfy $c_{i}(e) \leqslant C_{i} / n$ for $i=1,2, \ldots, r$. Let

$$
p=\mathbb{P}\left(c_{i}(e) \leqslant \frac{C_{i}}{n}, i=1,2, \ldots, r\right)=\prod_{i=1}^{r}\left(1-\exp \left\{-\left(\frac{C_{i}}{n}\right)^{1 / \beta}\right\}\right)
$$

and note that

$$
\frac{\log n}{n} \ll \frac{\Upsilon^{1 / \beta}}{2^{r} n^{r / \beta}} \leqslant p \leqslant \frac{\Upsilon^{1 / \beta}}{n^{r / \beta}} .
$$

The approach for this and the remaining problems is

(i) Look for a small weight structure in an edge weighted random graph $G$. In this case the random bipartite graph $G_{n, n, p}$. 
(ii) Use an idea of Walkup [15] to construct a random subgraph $H$ of $G$ that only uses edges of low weight.

(iii) Use a result from the literature that states that w.h.p. the edges of $H$ contain a copy of the desired structure.

$G$ is distributed as $G=G_{n, n, p}$. Note that by construction, a perfect matching $M$ of $G$ satisfies $c_{i}(M) \leqslant C_{i}, i=1,2, \ldots, r$.

Let $d=n p$ and note that because $d n p \gg \log n$ the Chernoff bounds imply that w.h.p. every vertex has degree $\approx d$. Now each edge of $G$ has a weight uniform in $[0,1]$. Following Walkup [15] we replace $w(e), e=(x, y)$ by $\min \left\{Z_{1}(e), Z_{2}(e)\right\}$ where

$$
Z_{1}, Z_{2} \text { are independent copies of } Z_{W} \text { where } \mathbb{P}\left(Z_{W} \geqslant x\right)^{2}=\mathbb{P}\left(Z_{E}^{\alpha} \geqslant x\right) \text {. }
$$

We assign $Z_{1}(e)$ to $x$ and $Z_{2}(e)$ to $y$.

Let $X, Y$ denote the bipartition of the vertices of $G$. Now consider the random bipartite graph $H$ where each $x \in X$ is incident to the two $Z_{1}$-smallest edges incident with $x$. Similarly, $y \in Y$ is incident to the two $Z_{2}$-smallest edges incident with $y$. Walkup [16] showed that $H$ has a perfect matching w.h.p. The expected weight of this matching is asymptotically at most

$$
\left(\frac{2^{\alpha} n}{d^{\alpha}}\right)\left(\Gamma\left(1+\frac{1}{\alpha}\right)+\Gamma\left(2+\frac{1}{\alpha}\right)\right) \times \frac{1}{2}=O\left(\frac{n^{1+r \alpha / \beta-\alpha}}{\Upsilon^{\alpha / \beta}}\right) .
$$

This follows from (i) the expression given in Corollary 6 for the expected minimum and second minimum of $d$ copies of $Z$ and (ii) the matching promised in [16] is equally likely to select a minimum or a second minimum weight edge.

The selected matching is the sum of independent random variables with exponential tails and so will be concentrated around its mean.

\subsection{Upper Bound for CMP}

We let $p, d$ be as in Section 4.1. We replace Walkup's result [16] by Frieze's result [8] that the random graph $G_{2-o u t}$ contains a perfect matching w.h.p. The random graph $G_{k-\text { out }}$ has vertex set $[n]$ and each vertex $v \in[n]$ independently chooses $k$ random edges incident with $v$. We again replace $c(e), e=(x, y)$ by $\min \left\{Z_{1}(e), Z_{2}(e)\right\}$ where $Z_{1}, Z_{2}$ are independent copies of $Z_{W}$ and associate one copy with each endpoint of the edge. We consider the random graph $H$ where each $v \in[n]$ is incident to the two $Z_{W}$-smallest edges incident with $x$. This is distributed as $G_{2-o u t}$ and we obtain an expression similar to that in (30).

We have concentration around the mean as in Section 4.1.

\subsection{Upper bound for CTSP+}

We first consider the TSP. For the symmetric case, we replace the weight $w(e), e=\{x, y\}$ by $\min \left\{Z_{1}(e), Z_{2}(e)\right\}$ for each edge of $K_{n}$ and for the asymmetric case we replace $w(e), e=$ 
$(x, y)$ by $\min \left\{Z_{1}(e), Z_{2}(e)\right\}$ for each directed edge of $\vec{K}_{n}$. In both cases we associate one copy of $Z_{W}$ to each endpoint of $e$. We define $p, d$ as in Section 4.1 and consider either the random graph $G_{n, p}$ or the random digraph $D_{n, p}$.

For the symmetric case, we consider the random graph $H$ that includes the 3 cheapest edges associated with each vertex, cheapest with respect to $Z_{W}(e)$. This will be distributed as $G_{3-\text { out }}$ which was shown to be Hamiltonian w.h.p. by Bohman and Frieze [4]. For the asymmetric case, we consider the random digraph $H$ that includes the 2 cheapest outedges and the 2 cheapest in edges associated with each vertex, cheapest with respect to $Z_{W}(e)$. This will be distributed as $D_{2-i n, 2-o u t}$ which has vertex set $[n]$ and where each vertex $v$ independently chooses 2 out- and in-neighbors. The random digraph $D_{2-i n, 2-o u t}$ was shown to be Hamiltonian w.h.p. by Cooper and Frieze [5].

The expected weight of the tour promised by [4] or by [5] is asymptotically equal to $O\left(n^{1+r \alpha / \beta-\alpha} / \Upsilon^{\alpha / \beta}\right)$ as in Section 4.1. We have concentration around the mean as in Section 4.1.

We obtain an upper bound for the MST through the fact that a Hamilton path is also a spanning tree. The same is true for the asymmetric case, since an arborescence is a tree with edges oriented away from the root.

\section{Lower Bounds}

We proceed as in Section 3.4. Suppose that $\Upsilon=\omega n^{r / \beta-1} \log n$ and $L=\frac{\varepsilon n^{1+r \alpha / \beta-\alpha}}{\Upsilon^{\alpha / \beta}}$ where $\varepsilon$ will be a sufficiently small constant. Let $\Lambda$ denote the relevant structure, matching or cycle. Then, by the union bound and Lemma 7, we have for CAP,CSTSP,CATSP, MST, MSA

$$
\begin{array}{r}
\mathbb{P}\left(\exists \Lambda: w(\Lambda) \leqslant L \text { and } c_{i}(\Lambda) \leqslant C_{i}, i=1,2, \ldots, r\right) \leqslant n^{n} \cdot \frac{L^{n / \alpha}}{\alpha^{n} n ! n^{n(1 / \alpha-1)}} \cdot \prod_{i=1}^{r} \frac{C_{i}^{n / \beta}}{\beta^{n} n ! n^{n(1 / \beta-1)}} \\
\leqslant\left(\frac{e \varepsilon^{1 / \alpha} n^{1 / \alpha+r / \beta-1}}{\alpha n^{1 / \alpha-1} \Upsilon^{1 / \beta}} \cdot \frac{e^{r} \Upsilon^{1 / \beta}}{\beta n^{r / \beta}}\right)^{n}=o(1),
\end{array}
$$

for $\varepsilon$ sufficiently small.

For CMP, assuming that $n=2 m$,

$$
\begin{aligned}
\mathbb{P}(\exists \Lambda: w & \left.\leqslant L \text { and } c_{i}(\Lambda) \leqslant C_{i}, i=1,2, \ldots, r\right) \\
& \leqslant \frac{n !}{m ! 2^{m}} \cdot \frac{L^{m / \alpha}}{\alpha^{m} m ! m^{m(1 / \alpha-1)}} \cdot \prod_{i=1}^{r} \frac{C_{i}^{m / \beta}}{\beta^{m} m ! m^{m(1 / \beta-1)}} \\
& \leqslant\left(\frac{\varepsilon^{1 / \alpha} m^{1 / \alpha+r / \beta-1}}{2 \alpha m^{1 / \alpha-1} \Upsilon^{1 / \beta}} \cdot \frac{e^{r} \Upsilon^{1 / \beta}}{\beta m^{r / \beta}}\right)^{m}=o(1),
\end{aligned}
$$

for $\varepsilon$ sufficiently small. 


\section{More general distributions}

We follow an argument from Janson [13]. We will asssume that $w(e)$, has the distribution function $F_{w}(t)=\mathbb{P}(X \leqslant t)$, of a random variable $X$, that satisfies $F_{w}(t) \approx a t^{1 / \alpha}, \alpha \leqslant 1$ as $t \rightarrow 0$. For the costs $c_{i}(e)$ we have $F_{c}(t) \approx b t^{1 / \beta}, \beta \leqslant 1$. The constants $a, b>0$ can be dealt with by scaling and so we assume that $a=b=1$ here. For a fixed edge and say, $w(e)$, we consider random variables $w_{<}(e), w_{>}(e)$ such that $w_{<}(e)$ is distributed as $Z_{E}^{\alpha+\varepsilon_{n}}$ and $w_{>}(e)$ is distributed as $Z_{E}^{\alpha-\varepsilon_{n}}$, where $\varepsilon_{n}=1 / 10 \log n$. (This choice of $\varepsilon_{n}$ means that $n^{\alpha+\varepsilon_{n}}=e^{1 / 10} n^{\alpha}$.) Then let $U(e)$ be a uniform $[0,1]$ random variable and suppose that $X$ has the distribution $F^{-1}(U)$. We couple $X, w_{<}, w_{>}$by generating $U(e)$ and then $w_{<}(e)=F_{<}^{-1}(U)=\log \left(\frac{1}{1-u}\right)^{\alpha-\varepsilon_{n}}$ and $F_{>}$is defined similarly. The coupling ensures that $w_{<}(e) \leqslant w(e) \leqslant w_{>}(e)$ as long as $w(e) \leqslant \varepsilon_{n}$.

Given the above set up, it only remains to show that w.h.p. edges of length $w(e)>\varepsilon_{n}$ or cost $c_{i}(e)>\varepsilon_{n}$ are not needed for the upper bounds proved above. We can ignore the lower bounds, because they only increase if we exclude long edges.

Assumptions for CMWP. For the minimum weight path problem we will assume that $\Upsilon^{1 / \beta} \gg \frac{\log ^{1+r / \beta} n}{n}$, which is a $\log n$ factor larger than required for Theorem 1 . We will assume that $C_{i}=o(1)$ and then we only use edges of cost of order $C_{i} / \log n \ll \varepsilon_{n}$.

Observe that the minimum weight of a path from 1 to $n$ is at most $\frac{4 \log n}{n p}$ w.h.p. and this is less than $\varepsilon_{n}$ because of the assumption $\frac{\log ^{1+r / \beta} n}{n} \ll \Upsilon^{1 / \beta}$ and the definition of $p$ (see $(2))$.

Assumptions for the other problems, We deal with costs by assuming that $C_{i}=$ $o(n / \log n), i=1,2, \ldots r$. It is then a matter of showing that w.h.p. the first few order statistics of $Z_{W}$ are very unlikely to be greater than $\varepsilon_{n}$. ( $Z_{W}$ is defined in (29).) But in all cases this can be bounded as follows: let $W_{1}, W_{2}, \ldots, W_{m}, m \geqslant n / 2$ be independent copies of $Z_{W}$. Then,

$$
\mathbb{P}\left(\left|\left\{i: W_{i} \leqslant \varepsilon_{n}\right\}\right| \leqslant 3\right) \leqslant m^{3}\left(1-\left(1-e^{-\varepsilon_{n}^{1 / \alpha}}\right)^{1 / 2}\right)^{m-3}=m^{3} e^{-m^{1-o(1)}} .
$$

This bounds the probability of using a heavy edge at any one vertex and inflating by $n$ gives us the result we need.

\section{Conclusion}

We have given upper and lower bounds that hold w.h.p. for constrained versions of some classical problems in Combinatorial Optimization. They are within a constant factor of one another, unlike the situation with respect to spanning trees and arborescences, [9], [10], where the upper and lower bounds are asymptotically equal. It is a challenge to find tight bounds for the problems considered in this paper and to allow correlation between length and cost. It would also be interesting to determine how feasiblity depends on $C_{1}, C_{2}, \ldots, C_{r}$. This presumably involves lower bounds on their values. If the $C_{i}$ are very large then w.h.p. they do not affect the optimum solution. The natural question is as to 
how large can they be so that the constraints are tight and yet very simple algorithms suffice to solve the optimisation problem.

We have not made any claims about $\mathbb{E}\left(w^{*}(\mathbf{C})\right)$ because there is always the (small) probability that the problem is infeasible. It is not difficiult to similarly bound the expectation conditional on feasibility.

\section{References}

[1] S. Arora, A.M. Frieze and H. Kaplan, A New Rounding Procedure for the Assignment Problem with Applications to Dense Graph Arrangement Problems, Mathematical Programming A 92 (2002) 1-36.

[2] S. Bhamidi and R. van der Hofstad, Weak disorder asymptotics in the stochastic mean-field model of distance, The Annals of Applied Probability 22 (2010) 1907-1965.

[3] S. Bhamidi and R. van der Hofstad, Weak disorder asymptotics in the stochastic mean-field model of distance II, Bernoulli 19 (2013) 363-386.

[4] T. Bohman and A.M. Frieze, Hamilton cycles in 3-out, Random Structures and Algorithms 35 (2009) 393-417.

[5] C. Cooper and A.M. Frieze, Hamilton cycles in random graphs and directed graphs, Random Structures and Algorithms 16 (2000) 369-401.

[6] E. Dijkstra, A note on two problems in connexion with graphs, Numerische Mathematik 1 (1959) 269-271.

[7] A.M. Frieze, On the value of a random minimum spanning tree problem, Discrete Applied Mathematics 10 (1985) 47-56.

[8] A.M. Frieze, Maximum matchings in a class of random graphs, Journal of Combinatorial Theory B 40 (1986) 196-212.

[9] A.M. Frieze and T. Tkocz, A randomly weighted minimum spanning tree with a random cost constraint, arXiv:1905.01229.

[10] A.M. Frieze and T. Tkocz, A randomly weighted minimum arborescence with a random cost constraint, arXiv:1907.03375.

[11] R. Hassin and E. Zemel, On shortest paths with random weights, Mathematics of Operations Research 10 (1985) 557-564.

[12] W. Hoeffding, Probability inequalities for sums of bounded random variables, Journal of the American Statistical Association 58 (1963) 13-30.

[13] S. Janson, One, two and three times $\log n / n$ for paths in a complete graph with random weights, Combinatorics, Probability and Computing 8 (1999) 347-361.

[14] R. Karp and M. Steele, R.M. Karp and J.M. Steele, Probabilistic analysis of heuristics, in The traveling salesman problem: a guided tour of combinatorial optimization, E.L. Lawler, J.K. Lenstra, A.H.G. Rinnooy Kan and D.B. Shmoys Eds. (1985) 181206. 
[15] D.W. Walkup, On the expected value of a random asignment problem, SIAM Journal on Computing 8 (1979) 440-442.

[16] D.W. Walkup, Matchings in random regular bipartite graphs, Discrete Mathematics 31 (1980) 59-64.

\section{A Auxilliary Lemmas}

Lemma 5. Let $\alpha>0$ and let $Y_{1}, Y_{2}, \ldots$ be i.i.d. copies of $Z=Z_{E}^{\alpha}$. For a positive integer $m$ and $1 \leqslant k \leqslant m$, let $X_{m}^{(k)}$ be the $k$ th minimum of $Y_{1}, \ldots, Y_{m}$. Then

$$
\mathbb{E} X_{m}^{(k)}=\Gamma(1+\alpha) \sum_{j=0}^{k-1} \sum_{i=0}^{j}\left(\begin{array}{c}
m \\
j
\end{array}\right)\left(\begin{array}{l}
j \\
i
\end{array}\right)(-1)^{i}(m+i-j)^{-\alpha} .
$$

In particular, if $k$ is a constant as $m \rightarrow \infty$, then

$$
\mathbb{E} X_{m}^{(k)} \approx \frac{1}{(k-1) !} \Gamma\left(k+\frac{1}{\alpha}\right) m^{-\alpha}
$$

Proof. Note that

$$
\mathbb{P}\left(X_{m}^{(k)}>t\right)=\sum_{j=0}^{k-1}\left(\begin{array}{c}
m \\
j
\end{array}\right) \mathbb{P}\left(Y_{1} \leqslant t\right)^{j} \mathbb{P}\left(Y_{1}>t\right)^{n-j}
$$

(for the $k$ th minimum to be larger than $t$, we need exactly $j$ variables to be at most $t$ and $m-j$ larger than $t, j=0,1, \ldots, k-1)$. Integrating gives

$$
\mathbb{E} X_{m}^{(k)}=\int_{0}^{\infty} \mathbb{P}\left(X_{m}^{(k)}>t\right) \mathrm{d} t=\sum_{j=0}^{k-1}\left(\begin{array}{c}
m \\
j
\end{array}\right) \int_{0}^{\infty}\left(1-e^{-t^{\alpha}}\right)^{j} e^{-(m-j) t^{\alpha}} \mathrm{d} t .
$$

It remains to expand $\left(1-e^{-t^{\alpha}}\right)^{j}$ and use $\int_{0}^{\infty} e^{-\lambda t^{\alpha}} \mathrm{d} t=\Gamma(1+\alpha) \lambda^{-\alpha}$. The asymptotic statements follow by writing $(m+i-j)^{-\alpha}=m^{-\alpha}\left(1+\frac{i-j}{m}\right)^{-\alpha}$ and applying the binomial series.

Corollary 6. Let $\alpha>0$ and let $\widehat{Y}_{1}, \widehat{Y}_{2}, \ldots$ be i.i.d. copies of $Z_{1}$, where $Z_{1}$ is as defined in (29). For a positive integer $m$ and $1 \leqslant k \leqslant m$, let $\widehat{X}_{m}^{(k)}$ be the $k$ th minimum of $\widehat{Y}_{1}, \ldots, \widehat{Y}_{m}$. Then

$$
\mathbb{E} \widehat{X}_{m}^{(k)}=2^{\alpha} \Gamma(1+\alpha) \sum_{j=0}^{k-1} \sum_{i=0}^{j}\left(\begin{array}{c}
m \\
j
\end{array}\right)\left(\begin{array}{l}
j \\
i
\end{array}\right)(-1)^{i}(m+i-j)^{-\alpha} .
$$

In particular, if $k$ is a constant as $m \rightarrow \infty$, then

$$
\mathbb{E} \widehat{X}_{m}^{(k)} \approx 2^{\alpha} \frac{1}{(k-1) !} \Gamma(k+\alpha) m^{-\alpha}
$$


Proof. This follows from Lemma 5 and the fact that $Z_{1}$ has the same distribution as $2^{\alpha} Z$ because we have $\mathbb{P}\left(Z_{1} \geqslant x\right)=\mathbb{P}(Z \geqslant x)^{1 / 2}=\mathbb{P}\left(2^{\alpha} Z \geqslant x\right)$. (Here $Z=Z_{E}^{\alpha}$.)

Lemma 7. Let $\alpha \leqslant 1$ and let $Y_{1}, Y_{2}, \ldots$ be i.i.d. copies of $Z_{E}^{\alpha}$. Then for $t \geqslant 0$, we have

$$
\mathbb{P}\left(Y_{1}+\cdots+Y_{n} \leqslant t\right) \leqslant \frac{t^{n / \alpha}}{\alpha^{n} n ! n^{n(1 / \alpha-1)}} .
$$

Proof. Using the density,

$$
\mathbb{P}\left(Y_{1}+\cdots+Y_{n} \leqslant t\right)=\int_{x_{1}, \ldots, x_{n} \geqslant 0, \sum x_{i} \leqslant t} \prod_{i=1}^{n} \alpha^{-1} x_{i}^{1 / \alpha-1} e^{-x_{i}^{1 / \alpha}} \mathrm{d} x_{1} \ldots \mathrm{d} x_{n} .
$$

By the AM-GM inequality,

$$
\prod_{i=1}^{n} x_{i} \leqslant\left(\frac{\sum_{i=1}^{n} x_{i}}{n}\right)^{n}
$$

and trivially $e^{-x_{i}^{1 / \alpha}} \leqslant 1$, so the integrand can be pointwise bounded as follows

$$
\prod_{i=1}^{n} \alpha^{-1} x_{i}^{1 / \alpha-1} e^{-x_{i}^{1 / \alpha}} \leqslant \alpha^{-n}\left(\frac{\sum_{i=1}^{n} x_{i}}{n}\right)^{n(1 / \alpha-1)} \leqslant \alpha^{-n} \frac{t^{n(1 / \alpha-1)}}{n^{n(1 / \alpha-1)}}
$$

Thus,

$\mathbb{P}\left(Y_{1}+\cdots+Y_{n} \leqslant t\right) \leqslant \alpha^{-n} \frac{t^{n(1 / \alpha-1)}}{n^{n(1 / \alpha-1)}} \cdot \operatorname{vol}\left\{x_{1}, \ldots, x_{n} \geqslant 0, \sum_{i=1}^{n} x_{i} \leqslant t\right\}=\alpha^{-n} \frac{t^{n / \alpha}}{n ! n^{n(1 / \alpha-1)}}$. 